\title{
Fatty acids and fatty aldehydes of buffalo seminal plasma and sperm lipid
}

\author{
Y. C. Jain and S. R. Anand \\ National Dairy Research Institute, Karnal-13200I, India
}

\begin{abstract}
Summary. Analysis of the fatty acids of total and neutral lipids, glycolipids, phospholipids and gangliosides of buffalo spermatozoa and seminal plasma showed that there were high levels of polyunsaturated acids. Neutral lipids were the richest in polyunsaturated acids $(55 \%$ in spermatozoa and $61 \%$ in seminal plasma). The major saturated acid of all the principal classes was stearic acid and the major unsaturated acid was docosahexaenoic acid $(22: 6 \omega 3)$ except in the neutral lipids in which it was arachidonic acid (20:4c06). The major aldehyde was palmitaldehyde $(16: 0)$ in buffalo sperm lipids and docosanal $(22: 0)$ in seminal plasma. More than $50 \%$ of the total aldehydes was contributed by aldehydes with a chain length greater than 18 carbon atoms.
\end{abstract}

\section{Introduction}

The fatty acid and fatty aldehyde composition of lipids of spermatozoa and seminal plasma have been examined in the bull, ram, boar, stallion, dog, rabbit, fowl and man (Flipse \& Potter, 1955; Scott, White \& Annison, 1961; Dietz, Pickett, Komarek \& Jensen, 1963; Pursel \& Graham, 1967; Ahluwalia \& Holman, 1969; Johnson, Gerrits \& Young, 1969; Poulos, Darin-Bennett \& White, 1973; Darin-Bennett, Poulos \& White, 1974) and fatty acid composition has usually been given of total semen, total phospholipids or of some component neutral lipid or phospholipid. Precise information about the fatty acid composition of the principal lipid classes and the various component lipids in each class has not been previously available. In the present study, buffalo semen was analysed because of the importance of these compounds in endogenous respiration and as components of membrane structures.

\section{Materials and Methods}

\section{Materials}

Boron trifluoride-methanol reagent was purchased from E. Merck, Darmstadt, Germany. Diethyleneglycol succinate (DEGS) and diatoport were the products of F \& M Corporation, Pennsylvania, U.S.A. Methyl esters of fatty acids were purchased from the Biochemical Unit, V.P. Chest Institute, Delhi, India or from Sigma, St Louis, U.S.A. The fatty aldehydes of different chain length were synthesized from fatty acids by the method of Celleja \& Rogers (1970).

\section{Semen collection and extraction of lipids}

Semen was collected with an artificial vagina and fractionated into spermatozoa and seminal plasma by centrifugation. Lipids were extracted from the two fractions separately with chloroformmethanol $(2: 1 \mathrm{v} / \mathrm{v})$ according to the procedure of Folch, Lees \& Sloane-Stanley (1957). The total lipids were fractionated into the principal lipid classes and component lipids by silicic acid-column and thin-layer chromatography (Jain \& Anand, 1976). The sialoglycolipids were extracted from the upper Folch phase (Jain \& Anand, 1975). 


\section{Formation and analysis of methyl esters and dimethyl acetals}

Lipid samples dissolved in $0.5 \mathrm{ml}$ benzene were treated with $2.5 \mathrm{ml} \mathrm{BF}_{3}$-methanol reagent. The methyl esters and dimethyl acetals formed were separated from each other by the method of Farquhar (1972). The methyl esters were further purified by thin-layer chromatography with petroleum ether-ethylether-acetic acid (90:10:1 by vol.) as the developing solvent (Morrison \& Smith, 1964). The extracts were run on an F \& M Model 609 gas chromatograph (F \& M Corporation, Pennsylvania, U.S.A.) equipped with a hydrogen flame ionization detector. The $5 \mathrm{ft} \times \frac{1}{4}$ inch column was packed with $20 \%$ DEGS on diatoport $\mathrm{P}(60-80 \mathrm{mesh})$ and was kept isothermally at $195^{\circ} \mathrm{C}$. The fatty acid methyl esters and dimethyl acetals were eluted with nitrogen at a flow rate of $70 \mathrm{ml} / \mathrm{min}$. Peaks were identified by comparing the retention time of the component fatty acids and aldehydes with those of known standards. Peak area was measured by triangulation. The miscellaneous fatty acid fraction included lower chain and branched chain acids and acids higher than 24:0 because standards were not available. The fatty acids have been expressed in short-hand nomenclature, i.e. number of carbon atoms: number of double bonds, and the position of the double bond is designated by $\omega$.

\section{Results}

\section{Total lipids}

The fatty acid composition of the total lipids of buffalo spermatozoa and seminal plasma is presented in Table 1. Acids with a chain length shorter than 12 carbons were not determined. Buffalo spermatozoa contained a nearly equal distribution of saturated $(47.8 \%)$ and unsaturated $(49.8 \%)$ fatty acids; in seminal plasma the proportion was slightly in favour of unsaturated acids. The principal saturated fatty acid of sperm and seminal plasma lipids was stearic acid. In the unsaturated class, docosahexaenoic acid was the major fatty acid. More than $70 \%$ of the total unsaturated acids were polyunsaturated and were the derivatives of linoleic $(18: 2 \omega 6)$ and linolenic $(18: 3 \omega 3)$ acids. There were no detectable amounts of acids derived from oleic acid $(18: 1 \omega 9)$. Neutral lipids contained the most polyunsaturated fatty acids (Text-fig. 1).

Table 1. Fatty acid and fatty aldehyde composition (as $\%$ of the total peak area; mean \pm S.E.M. of three samples analysed in duplicate) of the lipids of buffalo spermatozoa and seminal plasma

\begin{tabular}{|c|c|c|c|c|}
\hline \multirow[b]{2}{*}{ Acid/aldehyde } & \multicolumn{2}{|c|}{ Spermatozoa } & \multicolumn{2}{|c|}{ Seminal plasma } \\
\hline & Fatty acid & Fatty aldehyde & Fatty acid & Fatty aldehyde \\
\hline $12: 0$ & $2 \cdot 8 \pm 1 \cdot 2$ & - & $2 \cdot 6 \pm 1 \cdot 9$ & - \\
\hline $12: 1$ & $0 \cdot 3 \pm 0 \cdot 1$ & - & $0.4 \pm 0.3$ & - \\
\hline $14: 0$ & $4.5 \pm 1.2$ & $7 \cdot 6 \pm 1 \cdot 1$ & $5 \cdot 6 \pm 0 \cdot 3$ & $2.0 \pm 0.5$ \\
\hline $14: 1$ & $0.5 \pm 0.2$ & 二 & $0.6 \pm 0.3$ & - \\
\hline $15: 0$ & - & $1.3 \pm 0.4$ & - & $0.9 \pm 0.5$ \\
\hline $16: 0$ & $10 \cdot 8 \pm 2 \cdot 3$ & $22.3 \pm 1.9$ & $10 \cdot 9 \pm 1 \cdot 0$ & $15.8 \pm 1.4$ \\
\hline $16: 1$ & $3.7 \pm 0.7$ & - & $1.4 \pm 0.6$ & - \\
\hline $17: 0$ & 二 & $9 \cdot 3 \pm 0 \cdot 6$ & - & $3 \cdot 7 \pm 0.2$ \\
\hline $18: 0$ & $16 \cdot 7 \pm 2 \cdot 0$ & $6.6 \pm 0.9$ & $15.9 \pm 1.4$ & $5 \cdot 6 \pm 0 \cdot 6$ \\
\hline $18: 1$ & $6.9 \pm 0.2$ & $6 \cdot 7 \pm 0 \cdot 6$ & $11 \cdot 0 \pm 1 \cdot 5$ & $2.4 \pm 0.7$ \\
\hline $18: 2$ & $5.3 \pm 0.5$ & - & $4 \cdot 7 \pm 1 \cdot 2$ & - \\
\hline $18: 3$ & $3.9 \pm 1 \cdot 5$ & - & $2.4 \pm 0.9$ & - \\
\hline $20: 0$ & $7 \cdot 3 \pm 1 \cdot 5$ & $21 \cdot 1 \pm 1 \cdot 0$ & $3 \cdot 8 \pm 1 \cdot 3$ & $18 \cdot 5 \pm 1 \cdot 2$ \\
\hline $20: 4$ & $9 \cdot 0 \pm 2 \cdot 4$ & - & $13 \cdot 5 \pm 2.8$ & - \\
\hline $22: 0$ & $1.7 \pm 0.6$ & $14 \cdot 1 \pm 1 \cdot 0$ & $1.2 \pm 0.8$ & $29 \cdot 6 \pm 2 \cdot 2$ \\
\hline $22: 6$ & $20 \cdot 2 \pm 2 \cdot 4$ & - & $17 \cdot 6 \pm 4 \cdot 6$ & - \\
\hline $24: 0$ & $4 \cdot 0 \pm 2 \cdot 3$ & $9 \cdot 6 \pm 0.5$ & $4 \cdot 1 \pm 1 \cdot 5$ & $8 \cdot 8 \pm 1 \cdot 0$ \\
\hline Miscellaneous & $2 \cdot 4 \pm 0.2$ & $1 \cdot 2 \pm 0 \cdot 1$ & $4 \cdot 1 \pm 1 \cdot 9$ & $12 \cdot 7 \pm 1 \cdot 6$ \\
\hline
\end{tabular}




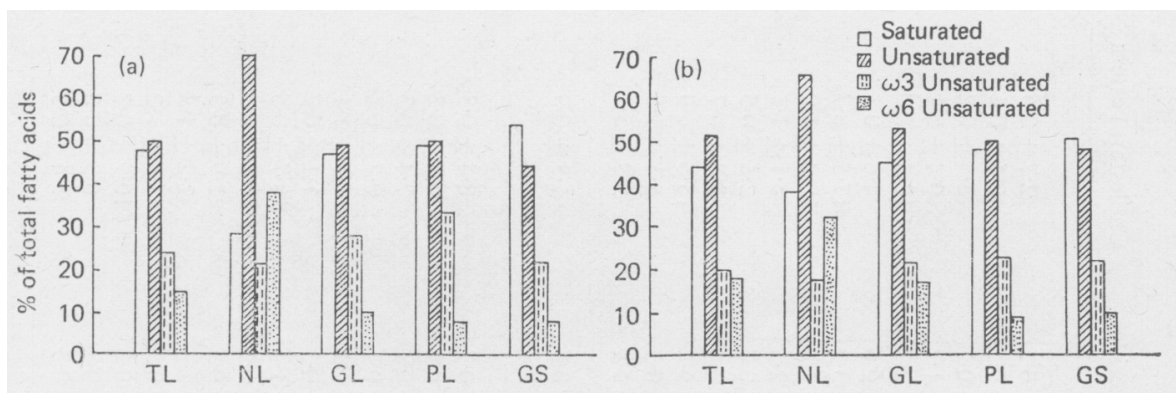

Text-fig. 1. The distribution of saturated, unsaturated and polyunsaturated acids derived from linoleic and linolenic acids in the total lipids (TL), neutral lipids (NL), glycolipids (GL), phospholipids (PL) and gangliosides (GS) of buffalo spermatozoa (a) and seminal plasma (b).

The fatty aldehyde composition of buffalo semen lipids (Table 1) revealed a wide range from $\mathrm{C}_{12}$ to $\mathrm{C}_{24}$. The most abundant aldehydes in the spermatozoa and seminal plasma were saturated, with more than $40 \%$ having a chain length greater than 20 carbons. The only unsaturated aldehyde found was $18: 1$.

\section{Neutral lipids}

The distribution of fatty acids in triglycerides (Table 2) was similar to that in the total neutral lipids. The content of polyunsaturated fatty acids was $54.6 \%$ in buffalo spermatozoa and $60.7 \%$ in seminal plasma triglycerides. Arachidonic acid constituted the major unsaturated fatty acid of the triglycerides, 1,2- and 1,3-diglycerides and cholesterol esters. Stearic acid was the principal saturated acid in all the neutral lipid classes except in the 1,2-diglycerides and cholesterol esters in which palmitic acid was found at slightly higher concentrations. The fatty acid profile in seminal plasma was qualitatively similar to that in spermatozoa but there were quantitative differences (Table 2).

\section{Phospholipids}

In the spermatozoa and seminal plasma, stearic acid was the major fatty acid of all the phospholipids except sphingomyelin, choline and ethanolamine plasmalogen and sperm phosphatidyl choline and ethanolamine (Table 3). In the last two fractions and sphingomyelin, docosahexaenoic acid occurred in the highest amounts but in choline and ethanolamine plasmalogen, oleic acid was the major fatty acid. Stearic acid was the principal saturated fatty acid in all the phospholipids and palmitic acid was the second principal acid. Docosahexaenoic acid was the major unsaturated acid in phosphatidic acid, sphingomyelin, phosphatidyl choline and ethanolamine, cardiolipin, sperm lysophosphatidyl ethanolamine and seminal plasma phosphatidyl inositol, and oleic acid the major unsaturated acid of lysophosphatidyl serine and choline, phosphatidyl serine, choline and ethanolamine plasmalogens, seminal plasma lysophosphatidyl ethanolamine and sperm phosphatidyl inositol. The ratio of unsaturated to saturated fatty acid was in favour of cardiolipin, choline and ethanolamine plasmalogens, sphingomyelin, sperm phosphatidyl choline and serine and seminal plasma phosphatidyl ethanolamine.

\section{Discussion}

Although Dietz et al. (1963) and Scott et al. (1961) did not report the presence of acids with a chain length greater than 18 carbon atoms, it is now clear that the semen of mammals contains a large proportion of polyunsaturated fatty acids. Ahluwalia \& Holman (1969) found 12-65\% polyunsaturated fatty acid in the total fatty acid content of sperm heads, tails and seminal plasma of 


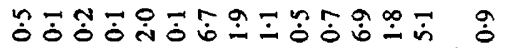
$+1+1+1+1+1+1+1+1+1+1+1+1+1+1 \mid+1$

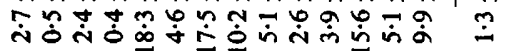

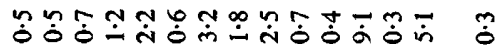
$+1+1+1+1+1+1+1+1+1+1+1+1+1+1 \mid+1$

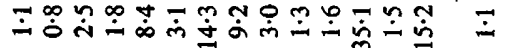

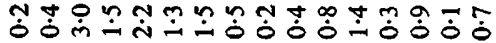
$+1+1+1+1+1+1+1+1+1+1+1+1+1+1+1+1$

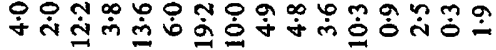

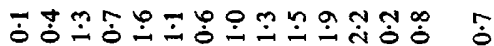
$+1+1+1+1+1+1+1+1+1+1+1+1+1+1 \mid+1$

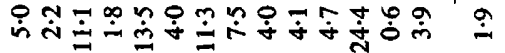

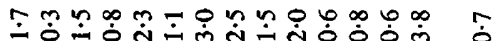
$+1+1+1+1+1+1+1+1+1+1+1+1+1+11+1$

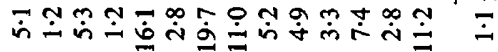

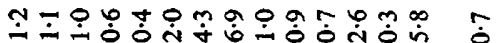
$+1+1+1+1+1+1+1+1+1+1+1+1+1+1 \mid+1$

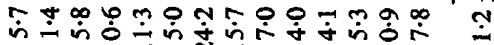

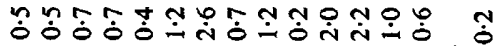
$+1+1+1+1+1+1+1+1+1+1+1+1+1+1 \mid+1$

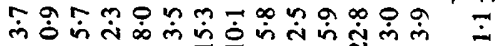

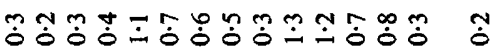
$+1+1+1+1+1+1+1+1+1+1+1+1+1+11+1$

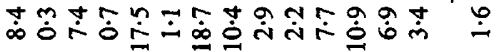

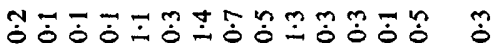
$+1+1+1+1+1+1+1+1+1+1+1+1+1+11+1$

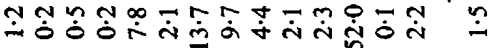

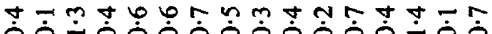
$+1+1+1+1+1+1+1+1+1+1+1+1+1+1+1+1$ $\infty$ NmNo

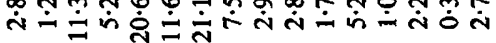

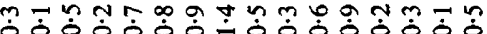
$+1+1+1+1+1+1+1+1+1+1+1+1+1+1+1+1$

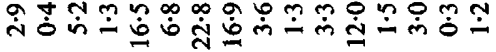

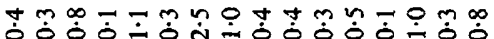
$+1+1+1+1+1+1+1+1+1+1+1+1+1+1+1+1$

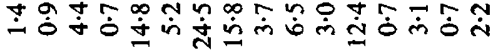

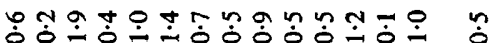
$+1+1+1+1+1+1+1+1+1+1+1+1+1+1 \mid+1$

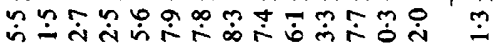

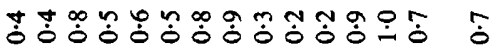
$+1+1+1+1+1+1+1+1+1+1+1+1+1+11+1$

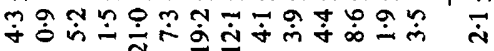

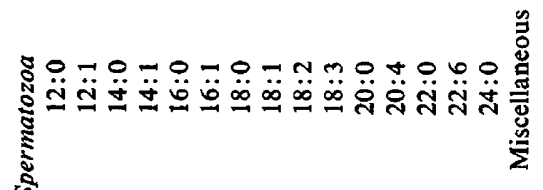

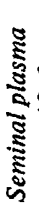




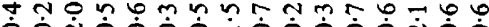
0 $\dot{0} \dot{0} 0 \dot{0} \dot{0} \dot{0} 0 \dot{0} 0 \dot{0} 0 \dot{0}$ $+1+1+1+1+1+1+1+1+1+1+1+1+1+1+1+1$

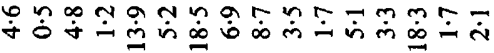

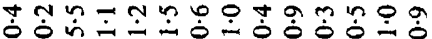
$+1+1+1+1+1+1+1+1+1+1+1+1+1+1$ ornon

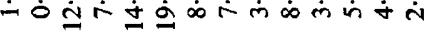

क் $+1+1+1+1+1+1+1+1+1+1+1+1+1+1+1+1$

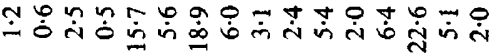

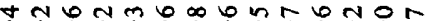
$\dot{0} \dot{0} \dot{-} \dot{-} \dot{0} \dot{0} \dot{0} \dot{\Delta} \dot{0} \dot{0} \dot{\sim} \dot{\mathrm{N}} \dot{0}$ $1+1+1+1+1+1+1+1+1+1+1+1+1+1+1$

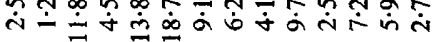

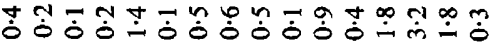
$+1+1+1+1+1+1+1+1+1+1+1+1+1+1+1+1$

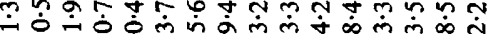

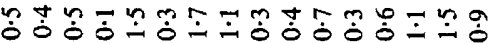
$+1+1+1+1+1+1+1+1+1+1+1+1+1+1+1+1$

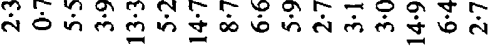

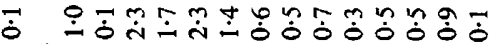
$+18+1+1+1+1+1+1+1+1+1+1+1+1+1+1$

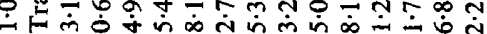

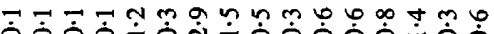
$+1+1+1+1+1+1+1+1+1+1+1+1+1+1+1+1$

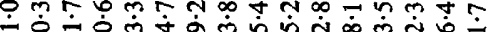

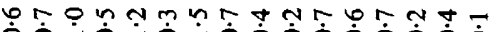
$7+1+1+1+1+1+1+1+1+1+1+1+1+1+1+1$

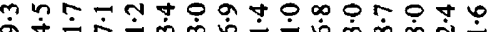

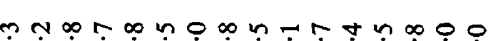
$\dot{0} \dot{0} \dot{0} \dot{0} \dot{0} \dot{m} \dot{0} \dot{0} \dot{-} \dot{-} \dot{0} \dot{0} \dot{-} \dot{-} \dot{0}$ $+1+1+1+1+1+1+1+1+1+1+1+1+1+1+1+1$

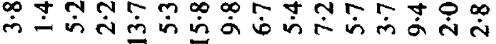

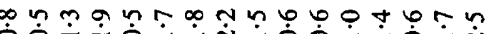
$+1+1+1+1+1+1+1+1+1+1+1+1+1+1+1+1$

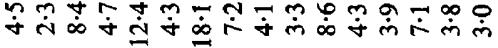

б̄ं் $+1+1+1+1+1+1+1+1+1+1+1+1+1+1+1+1$

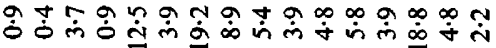

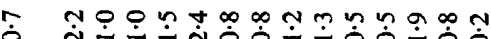
$\dot{0} \quad \dot{\sim} \dot{-} \dot{-} \dot{-} \dot{\sim} \dot{0} \dot{0} \dot{-} \dot{-} \dot{0} \dot{0} \dot{-} \dot{0} \dot{0}$

(1+1+1+1

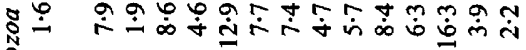

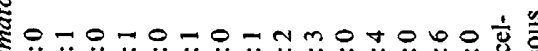

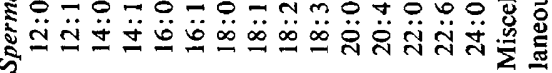

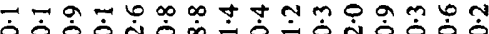

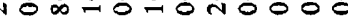
$+1+1+1+1+1+1+1+1+1+1+1+1+1+1+1+1$

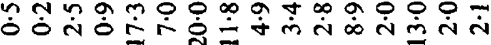

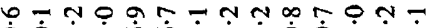
$\dot{0} \dot{0} \dot{-} \dot{0} \dot{0} \dot{-} \dot{-} \dot{0} \dot{0} \dot{\boldsymbol{\theta}} \dot{-} \dot{-}$ $1+1+1+1+1+1+1+1+1+1+1+1+1+1+1$

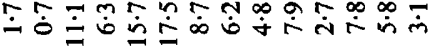

N-0.m $0 \quad \dot{0} \dot{-} \dot{0} \dot{\sim} \dot{0} \dot{0} \dot{0} \dot{0} \dot{0}$ $+11+1+1+1+1+1+1+1+1+1+1+1+1+1+1$

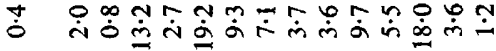

n n n n $1+1+1+1+1+1+1+1+1+1+1+1+1+1$

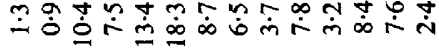

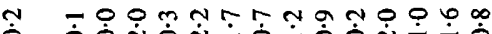
$+11+1+1+1+1+1+1+1+1+1+1+1+1+1+1$ サ

乌ீㅎํ் $+1+1+1+1+1+1+1+1+1+1+1+1+1+1+1+1$

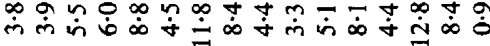

ஸ் $+1+1+1+1+1+1+1+1+1+1+1+1+1+1+1+$

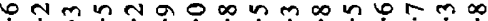

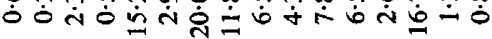

$0 \div n$ $\begin{array}{llll}+1+1+1+1+1+1+1+1+1+1+1+1+1+1+1+1 & 0\end{array}$ $+\infty N R a-n T m m m N a b-$

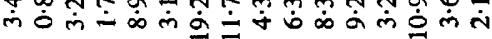

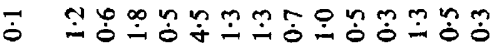
$+11+1+1+1+1+1+1+1+1+1+1+1+1+1+1$

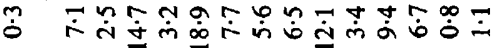

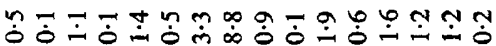
$+1+1+1+1+1+1+1+1+1+1+1+1+1+1+1+$

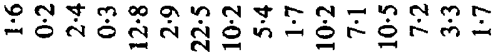

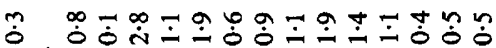
$+11+1+1+1+1+1+1+1+1+1+1+1+1+1+$ $0 \quad \forall N n \div N m n-n m n n \pi 0$

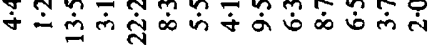

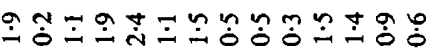
$81+1+1+1+1+1+1+1+1+1+1+1+1+1+$

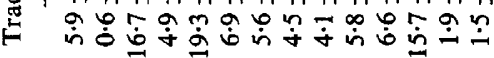

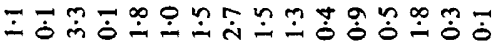
$8+1+1+1+1+1+1+1+1+1+1+1+1+1+1+1+$ हैo

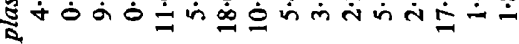

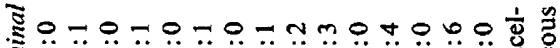

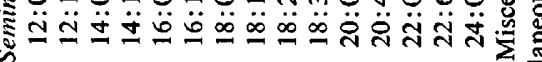


bull, boar, rabbit and man. Other studies on these and other species have yielded similar results. The high levels $(38 \%)$ in buffalo spermatozoa were due to the metabolites of linoleic and linolenic acids or their precursors. Unlike human semen (Ahluwalia \& Holman, 1969) in which almost all the known metabolites of linoleic and linolenic acids were present in measurable amounts, buffalo semen contained only arachidonic acid $(20: 4 \omega 6)$ and docosahexaenoic acid $(22: 6 \omega 3)$. Our results agreed qualitatively with those reported for ram and bull spermatozoa (Neill \& Masters, 1972, 1973) except that bulls also had small amounts of docosapentaenoic acid. Derivatives of oleic acid, though present in other reproductive tissues, were not detected in the seminal lipids of the animals studied by Ahluwalia \& Holman (1969) or in the buffalo.

Of the total unsaturated acids in the triglycerides, $55 \%$ in the spermatozoa $(38.1 \%$ of $\omega 6+16.5 \%$ of $\omega 3)$ and $61 \%$ in the seminal plasma $(56.4 \%$ of $\omega 6+4.3 \%$ of $\omega 3)$ were derivatives of linoleic and linolenic acids (Table 2). In the species studied by Ahluwalia \& Holman (1969), the $\omega 6$ acid content was $7 \cdot 5-33 \%$ and the $\omega 3$ acid $5 \cdot 3-28 \cdot 7 \%$. Although arachidonic acid was the major unsaturated acid of buffalo semen triglycerides, eicosapentaenoic acid $(20: 5 \omega 6)$ was reported to be so for man (Ahluwalia \& Holman, 1969). The presence of diglycerides but not free fatty acids has been reported in bull, ram, boar and stallion semen (Miller, Mayer \& Merilan, 1965; Komarek, Pickett, Lanz \& Jensen, 1964; Komarek, Pickett, Gibson \& Lanz, 1965; Komarek, Pickett, Gibson \& Jensen, 1965; Neill \& Masters, 1972, 1973). Buffalo spermatozoa and seminal plasma contained considerable amounts of both the lipids, indicating that lipolysis had occurred before the isolation of the lipids. However, a comparison of fatty acid composition of triglycerides or phospholipids with their hydrolysis products, diglycerides and free fatty acids showed no definite relationship. The phospholipids and triglycerides may not, therefore, be the substrates for the formation of diglycerides in buffalo semen although washed ram spermatozoa have been shown to form inositol phosphate from phosphatidyl inositol (Scott \& Dawson, 1968). Other pathways for the formation of diglycerides could be through the normal pathways involving phosphatidic acid intermediates (Kennedy, $1957)$ or through the acylation of monoglycerides (Clark \& Hubscher, 1961).

Docosahexaenoic acid was reported to be present in high amounts in bull sperm choline phosphoglycerides (Pursel \& Graham, 1967). Neill \& Masters $(1972,1973)$ showed that 22:6 was the principal acid of choline and ethanolamine phosphoglycerides in bull and ram sperm lipids. In the present study, choline and ethanolamine plasmalogens were separated from their phosphatidyl analogues and 22:6 was found to be the major fatty acid of phosphatidyl choline and phosphatidyl ethanolamine and oleic acid was the principal unsaturated acid of choline and ethanolamine plasmalogens. A similar distribution of fatty acids was observed in the four component phospholipids of buffalo seminal plasma but this differed from that found in other species (Pursel \& Graham, 1967; Ahluwalia \& Holman, 1969). The occurrence of palmitaldehyde and myristaldehyde as phospholipidbound aldehydes has been reported in bull, ram and boar spermatozoa, but studies on the detailed fatty aldehyde composition of semen phospholipids are few. Bull, ram and boar spermatozoa had at least $80 \%$ palmitaldehyde, while rabbit, human, dog and fowl spermatozoa contained relatively large amounts of stearaldehyde in addition to having palmitaldehyde as the predominant aldehyde (Poulos et al., 1973; Darin-Bennett et al., 1974). However, aldehydes with a chain length of 20 or more carbon atoms were not detected in these two studies. In the seminal lipids of buffalo, there were many aldehydes with a chain length of more than 18 carbons.

The high content of mono-, di- and trienoic fatty acids is characteristic of cardiolipin, which constitutes an important component of mitochondrial lipids (Stoffel, 1967). Bovine and ovine sperm cardiolipins have a high percentage of oleic and linoleic acids (Neill \& Masters, 1972, 1973); in buffalo semen cardiolipin there were more polyunsaturated fatty acids than monounsaturated acids.

We thank the Director, Dr D. Sundaresan, for encouragement and the Indian Council of Agricultural Research, New Delhi, for partly funding this work. One of us (Y.C.J.) was a recipient of a fellowship under the United Nations Development Programme. This paper is N.D.R.I. publication No. 75-163. 


\section{References}

Ahluwalia, B. \& Holman, R.T. (1969) Fatty acid composition of lipids of bull, boar, rabbit and human semen. J. Reprod. Fert. 18, 431-437.

Celleja, G.B. \& Rogers, P. (1970) A simple procedure for preparation and assay of small quantities of ${ }^{14} \mathrm{C}$-labelled long chain aliphatic aldehydes. $J$. Lab. Compounds 6, 135-142.

Clark, B. \& Hubscher, G. (1961) Direct esterification of monoglycerides in rat intestinal mucosa. Biochem. J. 80, 12P.

Darin-Bennett, A., Poulos, A. \& White, I.G. (1974) The phospholipid and phospholipid-bound fatty acids and aldehydes of $\operatorname{dog}$ and fowl spermatozoa. J. Reprod. Fert. 41, 471-474.

Dietz, R.W., Pickett, B.W., Komarek, R.J. \& Jensen, R.G. (1963) Fatty acid composition of bovine semen. J. Dairy Sci. 46, 468-472.

FARQUHAR, J.W. (1962) Identification and gas-liquid chromatographic behaviour of plasmalogen aldehydes and their acetal, alcohol, and acetylated alcohol derivatives. J. Lipid Res. 3, 210-30.

Flipse, R.J. \& Potter, E.F. (1955) Presence of fatty acids in bovine semen. Proc. Soc. exp. Biol. Med. 89, 432. 433 .

Folch, J., Lees, M \& Sloane-Stanley, G.H. (1957) A simple method for the isolation and purification of total lipids from animal tissues. J. biol. Chem. 226, 497-509.

JAIN, Y.C. \& ANAND, S.R. (1975) The occurrence of sialoglycolipids in bull, buffalo and goat semen. J. Reprod. Fert. 42, 129-132.

JAIN, Y.C. \& ANAND, S.R. (1976) The lipids of buffalo spermatozoa and seminal plasma. J. Reprod. Fert. 47, 255-260.

Johnson, L.A., Gerrits, R.J. \& Young, E.P. (1969) The fatty acid composition of porcine spermatozoa phospholipids. Biol. Reprod. 1, 330-334.

KenNedy, E.P. (1957) Metabolism of lipids. A. Rev. Biochem. 26, 119-142.
Komarek, R.J., Pickett, B.W., Lanz, R.N. \& Jensen, R.G. (1964) Lipid composition of bovine spermatozoa and seminal plasma. J. Dairy Sci. 47, 531536.

Komarek, R.J., PICkett, B.W., Gibson, E.W. \& JENSEN, R.G. (1965) Lipids of porcine spermatozoa, seminal plasma and gel.J. Reprod. Fert. 9, 131136.

Komarek, R.J., Pickett, B.W., Gibson, E.W. \& LANZ, R.N. (1965) Composition of lipids in stallion semen. J. Reprod. Fert. 10, 337-342.

Miller, L.D., Mayer, D.T. \& Merilan, C.P. (1965) Lipid classes of bovine spermatozoa. J. Dairy Sci. 48, 395-398.

MorRison, W.R. \& SMITH, L.M. (1964) Preparation of fatty acid methyl esters and dimethyl acetals from lipids with boron-trifluoride methanol. $J$. Lipid Res. 5, 600-608.

Neill, A.R. \& Masters, C.J. (1972) Metabolism of fatty acids by bovine spermatozoa. Biochem. $J$. 127, 375-385.

NeIll, A.R. \& Masters, C.J. (1973) Metabolism of fatty acids by ovine spermatozoa. J. Reprod. Fert. 34, 279-287.

Poulos, A., Darin-Bennett, A. \& White, I.G. (1973) The phospholipid bound fatty acid and aldehydes of mammalian spermatozoa. Comp. Biochem. Physiol. 46, 541-549.

Pursel, V.G. \& Graham, E.F. (1967) Phospholipid of bovine spermatozoa and seminal plasma. $J$. Reprod. Fert. 14, 203-211.

ScotT, T.W. \& Dawson, R.M.C. (1968) Metabolism of phospholipids by spermatozoa and seminal plasma. Biochem. J. 108, 457-463.

ScotT, T.W., White, I.G. \& Annison, E.F. (1961) Fatty acids in semen. Biochem. J. 78, 740-742.

Stoffel, W. (1967) The chemistry of mammalian lipids. In Lipid and Lipidases, pp. 1-39. Ed. G. Schettler. Springer-Verlag, Heidelberg.

Received 10 November 1975 